\title{
Evaluation of Immune Response in Mouse Blood Post Injection with Gamma Ray-Attenuated Sporozoites of Plasmodium berghei
}

Mukh Syaifudin $^{1}$, Darlina ${ }^{1}$, Tur Rahardjo ${ }^{1}$, Siti Nurhayati ${ }^{1}$, Devita Tetriana ${ }^{1}$, Zubaidah Alatas ${ }^{1}$, Puji Budi Setia Asih $^{2}$, Din Syafruddin ${ }^{2}$ and Rita Marleta Dewi ${ }^{3}$

1. Nuclear Medicine Technique and Radiation Biology Division, Center for Technology of Radiation Safety and Metrology, National Nuclear Energy Agency (BATAN), Lebakbulus 12440A, Jakarta, Indonesia

2. Malaria Group, Eijkman Institute for Molecular Biology, Ministry of Research and Technology, Salemba 10430, Jakarta, Indonesia

3. National Institute of Health Research and Development, Ministry of Health, Rawasari 10012, Jakarta, Indonesia

\begin{abstract}
Ionizing radiation has been successfully used to attenuate parasites for malaria vaccine development. To get a deeper insight in the immune response post injection of irradiated pre-erythrocytic stage of malaria parasites (sporozoites), we used Plasmodium berghei as a model. Anopheles sp. with infective parasites in their salivary glands was irradiated with 0-225 Gy of gamma rays. The isolated sporozoites were intravenously injected into groups of mouse followed by a booster and challenge in interval time of 2-4 weeks. The detection of parasite in the infected mosquito was done with nested-PCR (polymerase chain reaction). Immune response in mouse blood was examined with ELISA (enzime-linked immunosorbent assay) and protein profile of irradiated sporozoites was studied with SDS-PAGE (sodium dodecyl sulphate-polyacrylamide gel electrophoresis). By using Anopheles farauti as the more susceptible mosquito to parasite infection, based on molecular detection and parasites observation in mouse blood, there was low or no effect of irradiated sporozoites on the immune responses in mouse serum. Some factors affecting these results are discussed. There was a slight alteration of protein profile of sporozoite infected salivary glands post gamma ray irradiation except for its band intensity, indicating the low effectiveness of gamma irradiation at optimal dose. It can be concluded that irradiated sporozoite as vaccine materials was failed in eliciting immune response.
\end{abstract}

Key words: Gamma rays, malaria, P. berghei, sporozoites, vaccine materials.

\section{Introduction}

Malaria is still a major health problem in the world, causing high mortality mostly among children under the age of five years living in sub-Saharan Africa. More than 500 million people are infected by malaria and over one million of them die each year, accounts for $2.23 \%$ of deaths worldwide [1, 2]. In Indonesia, almost $45 \%$ of populations are having risk for malaria infection [3]. Indonesian Ministry of Health reported that the number of malaria cases dropped to

Corresponding author: Mukh Syaifudin, Ph.D., research field: radiation biology. E-mail: mukh_syaifudin@batan.go.id. over 1.1 million in 2009 from about 3 million per year in the past due to appropriate techniques in combating the deadly disease.

However, there are serious issues around the effective use of antimalarial drugs that include increasing multidrug resistance to $P$. falciparum and $P$. vivax. Consequently, a malaria vaccine that protects against infection and severe disease is urgently needed [4]. Therefore, research and development of a cost-effective deployable vaccine will be needed to facilitate elimination of malaria [5].

Research showed that the irradiated sporozoite remains the "gold standard" for the development of 
malaria vaccines that target the pre-erythrocytic stages of parasites [6]. Early studies demonstrated that experimental rodent hosts and human volunteers immunized with irradiated sporozoites developed antibodies that neutralized sporozoite infectivity [6-8]. These antibodies inhibit sporozoite motility, migration to the liver, and invasion of host hepatic cells $[9,10]$. Many preliminary vaccines have been based on the $P$. falciparum circumsporozoite surface antigen, circumsporozoite protein, or its epitopes because immune response of the body generally targets this protein when mounting an attack on the parasite [11]. The most promising being the demonstration that non immune volunteers repeatedly challenged and cured with pre-erythrocytic-stage parasites developed immunity to subsequent challenge as well as the demonstration of the efficacy of the first liver-stage vaccine tested in a malaria endemic area to reduce parasite density in children [12, 13].

Thus, the irradiated sporozoite vaccine is an excellent model for malaria vaccine development, because irradiated sporozoites enter hepatocytes and only partially develop within these cells. Such approaches have been repeated in mice using otherwise lethal infections of $P$. berghei $[14,15]$ and Plasmodium yoelii [16]. From these, a number of sporozoite surface antigens have already been identified and considered as vaccine candidates, but it is still in limited success. Here, we attempt to analyze the immunity in malaria by using $P$. berghei as a model. Due to protective immunity to parasitosis requires repeated, a booster was also done. Since vaccines rely strongly on stimulation of antibody responses, understanding the effects of infection on humoral immunity is an important step in effective vaccine development.

The objective of this study was to obtain the optimum irradiation dose delivered to parasites to attenuate and characterize all parameters required to obtain reliably malaria vaccine materials. We showed that irradiated parasite as vaccine material was not fully effective in eliciting immune response.

\section{Materials and Methods}

\subsection{Parasite and Mouse}

P. berghei ANKA (Antwerpen-Kasapa) strain infected mouse bloods were obtained from Eijkman Institute for Molecular Biology, Indonesian Ministry of Research and Technology. Male Swiss-Webster mice (6-8 weeks old) were purchased from Tropical Medicine Laboratory, National Institute of Health Research and Development, Indonesian Ministry of Health and were housed at the Biomedical Laboratory of The Center for Technology of Radiation Safety and Metrology, National Nuclear Energy Agency of Indonesia (BATAN) animal facility and handled according to institutional guidelines. All procedures were reviewed and approved by the Animal Care and Use National Commission, Institute of Health Research and Development, the Indonesian Ministry of Health.

\subsection{Anopheles sp. Rearing}

Anopheles maculates and An. farauti were reared at a temperature of around $26{ }^{\circ} \mathrm{C}$ and $70 \%$ relative humidity in the insectariant of the Center for Application of Isotope and Radiation, BATAN. For the selection experiment, 3-8 days old female mosquitoes were first separated out into groups of 100-200 individuals per cage. Experiments comprised of mosquitoes which were 1-3 days apart in age. Mosquitoes were maintained on distilled water for 12-15 hours prior to feeding on a restrained guinea for up to 45 minutes.

\subsection{Infection of Mouse}

Mice were IP (intraperitoneally) injected with parasitized mouse blood containing about $10^{6} \mathrm{P}$. berghei/mL. Parasitaemia was monitored started in the third day after infection by Giemsa-stained blood smears using light microscopy and was repeated every two days. When the parasitaemia was about 1\%-5\% (on day 5-7 of infection or gametocyte formation), 
mice were used for mosquito infection.

\subsection{Infection of Mosquito}

Standard protocols were used for infecting mosquitoes. It was done by feeding upon gametocyte-carrying 6-8 week old mice. Mosquitoes that had been starved overnight were placed into plastic containers (bugdorm). Hair of $P$. berghei infected mouse was removed from around $4 \mathrm{~cm}^{2}$ area of the back. Mosquitoes were allowed to feed on mice for 1-2 hours. After feeding, only fully engorged mosquitoes were kept up to 14-15 days securely in about $1 / 5$ liter cartoon cages, a cotton impregnated with a solution of sugar water was put on it and covered with a piece of net and placed in clipped transparent plastic completed with $50 \mathrm{~mL}$ centrifuge tube containing distilled water for humidity. Mosquitoes were maintained on glucose water soaked sterile cotton balls and changed every three days until dissection. The parasite infection status within mosquito was monitored at 8-9 days by examining 3-5 mosquito midguts for oocysts.

\subsection{Extraction of Mosquito DNA}

Head and thorax of mosquitoes were ground with teflon pestles in $1.5 \mathrm{~mL}$ Eppendorf microtubes containing $25 \mu \mathrm{L}$ of grinding buffer $(0.1 \mathrm{M} \mathrm{NaCl}, 0.2$ M sucrose, $0.1 \mathrm{M}$ Tris $\mathrm{HCl}, 0.05 \mathrm{M}$ EDTA, 0.5\% sodium dodecyl sulfate; $\mathrm{pH}$ adjusted to 9.2). Genomic nucleic acid was isolated from mosquito by the method as described in Ref. [17].

\subsection{Detection of Sporozoite in Mosquito with Nested-PCR}

The presence of sporozoite was assessed on days 14-15 by using PCR method. Oligonucleotide primers for PCR assay were designed based on the Plasmodium small subunit ribosomal RNA genes. Each $25 \mu \mathrm{L}$ reaction mixture for nest 1 amplifications contained $5 \mu \mathrm{L}$ of DNA template, $250 \mathrm{nM}$ of each primer (rPLU 1 and rPLU 5), $4 \mathrm{mM} \mathrm{MgCl}_{2}$, PCR buffer (50 $\mathrm{mM} \mathrm{KCl,}$ $10 \mathrm{mM}$ Tris-HCl), $200 \mathrm{mM}$ of each deoxynucleoside triphosphate, and 1.25 units of Taq DNA polymerase. Nested 1 amplification conditions were: initial denaturation at $94{ }^{\circ} \mathrm{C}$ for $5 \mathrm{~min}$, followed by 35 cycles that consists of denaturation at $94{ }^{\circ} \mathrm{C}$ for $30 \mathrm{sec}$; annealing at $55{ }^{\circ} \mathrm{C}$ for $1 \mathrm{~min}$; extension at $72{ }^{\circ} \mathrm{C}$ for 1 min; final elongation at $72{ }^{\circ} \mathrm{C}$ for $5 \mathrm{~min}$. Two microliters of the nested 1 amplification product served as the DNA template for each of the $20-\mu \mathrm{L}$ nested 2 amplifications. The concentration of the nested 2 primers and other constituents were identical to nested 1 amplifications except that 0.5 units of Taq DNA polymerase were used. Nested 2 amplification conditions were identical to those of nest 1 except that the extension at $72{ }^{\circ} \mathrm{C}$ was for 1 min and 30 seconds of 30 cyclus for the genus-specific primers (rPLU 3 and rPLU 4). The PCR products of nested 2 amplifications were analyzed by gel electrophoresis and stained with ethidium bromide.

\subsection{In vivo Irradiation and Isolation of Sporozoites}

Fourteen days after infective blood meal mosquitoes with the presence of sporozoites in their salivary glands were irradiated in vivo with doses of $0,100,125,150$, 175 and $200 \mathrm{~Gy}$ at a dose rate of $380.2 \mathrm{~Gy} /$ hour. Irradiation was done with gamma source of Cobalt-60 machine located at the Center for Application of Isotope and Radiation, National Nuclear Energy Agency. Dose rate of irradiation was determineded by Fricke dosimeter. Sporozoites for immunization were isolated by anesthetized mosquitoes on ice after the legs and wings were removed. Infected salivary glands were dissected out and triturated in saline solution $(0.9 \% \mathrm{NaCl})$, after which freed sporozoites were intravenously injected to 2-4 healthy mice. We used 10 pairs of salivary glands for each of mouse.

\subsection{First Injection, Booster and Challenge for Sporozoites}

For immunization, mouse received an initial injection of irradiated sporozoites. Intravenously injections into the tail of mouse were given in a volume 
of $50-100 \mu \mathrm{L}$ of saline solution containing salivary glands per mouse. This process was repeated for the booster immunization two weeks after the first injection. Some mice that were not treated for a booster acted as control. Mice were challenged in the same way by injection of non irradiated parasites with lower concentration (about half of immunization and booster) 2-4 weeks after booster. Some mice were not treated for a challenge. Thin blood smears were prepared from drops of tail blood on a glass slide starting on day 4 up to 14 days after challenge. Slides were air dried, fixed with $100 \%$ methanol and stained with $10 \%$ Giemsa for 10 minutes. The percentage of parasitized red blood cells was determined microscopically. The mice were sacrificed 2-4 weeks post challenge and blood was collected from the heart. Serum was isolated by centrifugation, and antibody levels were determined using ELISA (Enzyme linked immunosorbent assay) kit.

\subsection{ELISA}

Procedure for the determination of immune response in mouse serum with ELISA method was done according to standard protocol [18]. After coated with $50 \mathrm{uL}$ of antigens ( $\mathrm{Pv}$ (Plasmodium vivax) 247, Pv210 and Pf (P. falciparum)) at $10 \mathrm{mg} / \mathrm{mL}$ in coating buffer, the plate was kept at $4{ }^{\circ} \mathrm{C}$ overnight. Plate was blocked with $100 \mu \mathrm{L}$ of $0.5 \%$ BSA in coating buffer for 3-4 h at $37{ }^{\circ} \mathrm{C}$ and washed four times with washing buffer. Fifty microlitter of serum samples diluted 1:100 $(v / v)$ in blocking buffer was added and leaved them for $1 \mathrm{~h}$ at $37{ }^{\circ} \mathrm{C}$. After four times with washed buffer, $50 \mu \mathrm{L}$ of ALP-conjugated or biotinylated anti-Ig of appropriate specificity at the recommended concentration in Tween-buffer was added and leaved for $1 \mathrm{~h}$ at $37{ }^{\circ} \mathrm{C}$. The sample was washed four times with $0.9 \% \mathrm{NaCl}$ plus $\quad 0.05 \%$ Tween. Fifty microlitter of streptavidin—ALP diluted 1:2,000 in Tween-buffer was added and leaved the sample for $1 \mathrm{~h}$ at $37{ }^{\circ} \mathrm{C}$. The sample was washed four times with $0.9 \% \mathrm{NaCl}$ plus $0.05 \%$ Tween. The sample was developed with $50 \mu \mathrm{L}$ of NPP (1 tablet/5 mL of substrate buffer) and read at OD (optical density) $405 \mathrm{~nm}$. Positive signal cut-off was defined as two times the mean OD value from normal serum. Antibody titer was determined as the highest sample dilution which produced an OD value greater than or equal to the cut-off.

\subsection{Protein Profile Post Irradiation}

The procedure was done according to standard protocol [19]. Samples of mosquito salivary glands that were irradiated with 0, 150, 175 and $200 \mathrm{~Gy}$ of gamma rays were added with acetone and sonicated for 15 minutes, added with Laemli buffer solution, warmed in boiled water for five minutes, centrifuged at $8,000 \mathrm{rpm}$ for five minutes, and electrophoreted onto $12 \%$ SDS-polyacrylamide gel. A total volume of $15 \mu \mathrm{L}$ protein extract solution was loaded into each well and electrophoresis was carried out at $200 \mathrm{~V}$ until the bromophenol blue dye reaches $1 \mathrm{~cm}$ above the bottom of the gel. After stained with Commassie R-250 for $1 \mathrm{~h}$, gel was destained for 24 hours, and the appeared protein bands on gel were analyzed. As a molecular weight marker, SeeBluePlus (Invitrogen) was used.

\section{Results}

In the current study, we investigate the effectiveness of radiation-attenuated pre-erythrocytic parasites for protection against parasitemia and severe disease in experimental model of malaria. We used the highly virulent murine malaria parasite $P$. berghei ANKA which produces two distinct yet uniformly fatal pathologies and because its ability to sequester within the microcirculation which is the characteristic of severe malaria [7].

Our experiment was dependent on the sporozoites existence in salivary glands of mosquito. Mass production of sporozoites requires intensive production of mosquitoes and experimental infection of vector that is determined by the existence of gametocytes in mouse blood. Some factors interfering with breeding and experimental infection of this anopheline species. They 
are including insectarium temperature, pattern and rhythm of blood feeding, and parasite, host and mosquito parameters which interfere with the yield of experimental infections. The increase of temperature promotes growth of parasite, but it also enhances bacterial and mycosal pollution so that it reduces survival of mosquitoes [20]. A condition that should be addressed in sporozoite production is also the existence of oocyst in midgut of mosquito after infection. One experiment found the oocyst in midgut of An. farauti at day 8 after $P$. berghei infected blood meal (Fig. 1), indicating that An. farauti was the most susceptible mosquito to get sporozoites.

Several factors related to genetic and environmental affecting sporozoite production in anopheles mosquitoes mainly the genome numbers per oocyst such as the size of the mosquito and the number of oocysts on the midgut. Moreover, the potential of the mosquito to serve as a vector depends on the ability to support sporogony, mosquito abundance, and contact with humans, which are all influenced by climatic and ecological factors. The ability to support sporogony is largely dependent upon species in that not all species of Anopheles are susceptible to Plasmodium infection. Temperature and mosquito longevity are other key factors affecting the parasite's interaction with the vector. Temperature also affects the time of development in that the duration of sporogony is substantially shorter at higher temperatures. A shorter duration of sporogony increases the chances that the mosquito will transmit the infection within its lifespan [21].

It was found that vaccine materials created from irradiated sporozoite was not seen its full effectivity in eliciting immunity after injected into the mouse. Several factors may affect this finding, one of them was predicted that sporozoite suspensions used for immunization may heavily contaminated with mosquito components. There is an alternate approach by allowed irradiated mosquitoes to directly inoculate attenuated sporozoites into hosts, the mosquitoes thereby acting as vehicles of immunization. Beside that, it is known that a large number of irradiated sporozoites are required to elicit protection in host. Another reason is that there were very low parasites in blood of mouse after bitten by infected mosquitoes. This is mainly due to, in our opinion, low capacity of all species of mosquito used as malaria vector in this experiment (An. maculates and An. farauti). There was no infection found in sporozoite injected healthy mouse at days of obervation. This might be due to low number of sporozoite or no sporogoni processes taken place in the mosquitoes that may cause no transmission of infection from mouse-sporozoite-mouse cycle.

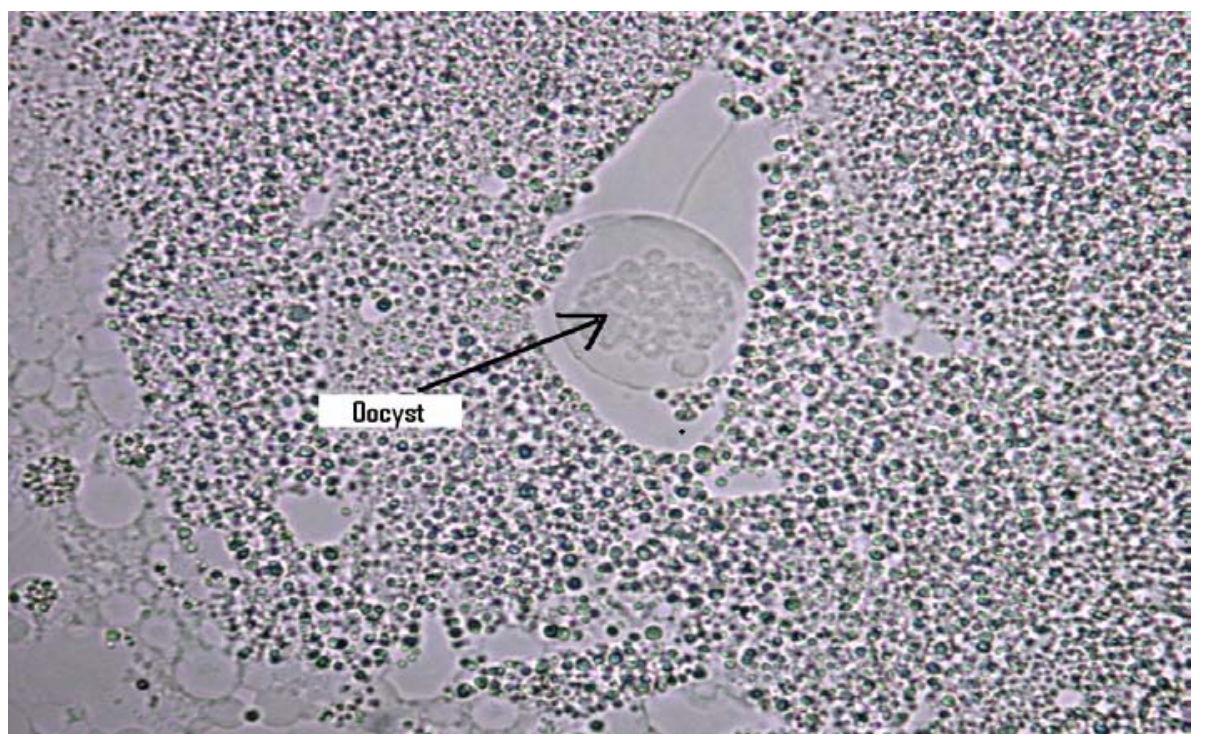

Fig. 1 Microscopic view of oocysts (arrow) in the midgut of P. berghei infected An. farauti at day 8 after infective blood meal. 
The detection of parasite in the mosquito with nested PCR by using the primers designed for the parasites characterization showed that An. farauti was more susceptible to parasite infection and supports the complete life cycle of $P$. berghei compared to An. maculates (Fig. 2), even though the number of sporozoites was very low to induce an infection in mouse. The mosquito species that mostly used in such studies is An. stephensi, which is robust mosquitoe considered to be highly efficient for sporozoite production [7, 22].

The observation of parasites in thin mouse blood smear showed that there was no parasite found in blood at days post challenge.

ELISA results presented in Table 1 shows that there was seemingly low effect of the booster and challenge on the positivity immune responses. However, there was tendency in increasing of the number of antigenic positive in serum samples with the increasing of irradiation doses mainly for 175 Gy. For 0, all samples (100\%) were positives in immune response, indicating that the immunity developed after one or two infections with infective parasites. For $100 \mathrm{~Gy}$,
50\%-75\% samples were positives, whereas for $125 \mathrm{~Gy}$ it was $0-50 \%$, and for 150,175 and $200 \mathrm{~Gy}$ there were $0-100 \%$ positive to Pf antigen. This also showing that Pf antigen was more recognized by mouse serum compared to Pv247 and Pv210 antigens that may be

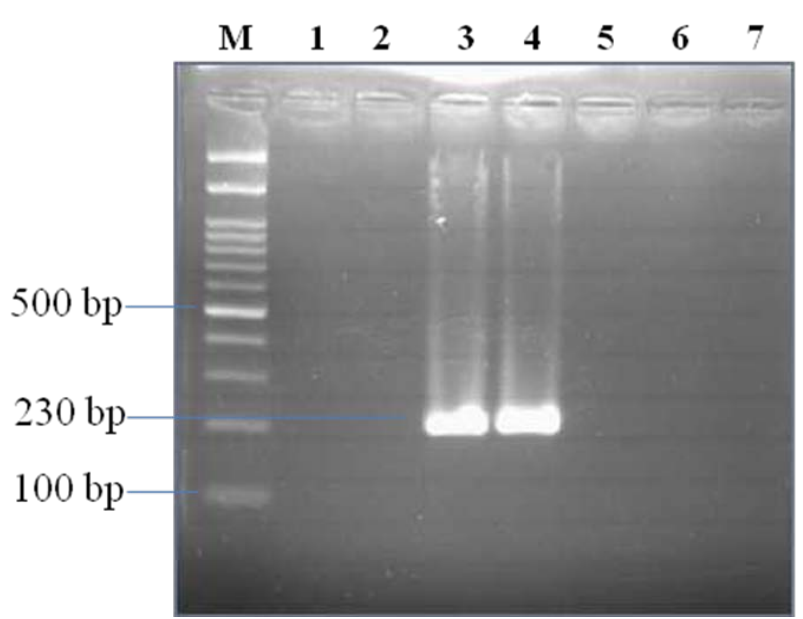

Fig. 2 The result of detection of parasite's DNA in the mosquito with nested PCR using the primers designed for the parasites characterization and 230 bp products. $M$, marker of 100 bp ladder; Lane 1, Anopheles maculates 1; lane 2, Anopheles maculates 2; lane 3, Anopheles farauti 1; lane 4, Anopheles farauti 2; lane 5, DNA of body of mosquitoes; lane 6, negative control for 1st PCR; lane 7, negative control for 2nd PCR.

Table 1 Results of ELISA on blood serum of mouse injected with irradiated sporozoite isolated from salivary glands of An. farauti, followed by booster and challenge.

\begin{tabular}{|c|c|c|c|c|c|c|}
\hline \multirow{2}{*}{$\begin{array}{l}\text { Irradiation } \\
\text { dose (Gy) }\end{array}$} & \multirow{2}{*}{$\begin{array}{l}\text { No. mouse } \\
\text { (sex; F: female; M: male) }\end{array}$} & \multirow{2}{*}{$\begin{array}{l}\text { Booster } \\
\text { (weeks post prime) }\end{array}$} & \multirow{2}{*}{$\begin{array}{l}\text { Challenge test (weeks post } \\
\text { booster) }\end{array}$} & \multicolumn{3}{|c|}{$\begin{array}{l}\text { No. positive samples to antigens of } \\
\text { ELISA/total }\end{array}$} \\
\hline & & & & Pv247 & Pv210 & Pf \\
\hline \multirow{3}{*}{0} & $2, \mathrm{~F}$ & 2 & - & 0 & 0 & $2 / 2$ \\
\hline & $2, \mathrm{M}$ & 2 & 4 & 0 & 0 & $2 / 2$ \\
\hline & $4, \mathrm{M}$ & - & - & 0 & 0 & $3 / 4$ \\
\hline 100 & $4, \mathrm{M}$ & - & - & 0 & 0 & $1 / 4$ \\
\hline \multirow{4}{*}{125} & $2, \mathrm{M}$ & - & 2 & 0 & 0 & $0 / 2$ \\
\hline & $2, \mathrm{M}$ & 2 & 4 & $1 / 2$ & 0 & $1 / 2$ \\
\hline & $2, \mathrm{M}$ & - & - & 0 & 0 & $2 / 2$ \\
\hline & $2, \mathrm{M}$ & - & 2 & 0 & 0 & $0 / 2$ \\
\hline \multirow[b]{2}{*}{150} & $2, \mathrm{M}$ & 2 & - & 0 & $1 / 2$ & $0 / 2$ \\
\hline & $2, \mathrm{M}$ & 2 & 2 & 0 & $1 / 2$ & $2 / 2$ \\
\hline \multirow{4}{*}{175} & 4, M & - & - & 0 & 0 & $3 / 4$ \\
\hline & $2, \mathrm{M}$ & - & 2 & 0 & 0 & $0 / 2$ \\
\hline & $2, \mathrm{~F}$ & 2 & 2 & 0 & 0 & $1 / 2$ \\
\hline & $2, \mathrm{M}$ & 2 & 4 & 0 & 0 & $2 / 2$ \\
\hline \multirow{3}{*}{200} & $2, F$ & 2 & - & 0 & 0 & $0 / 2$ \\
\hline & $4, \mathrm{M}$ & 2 & 2 & 0 & 0 & $0 / 4$ \\
\hline & $2, \mathrm{~F}$ & 2 & 4 & 0 & 0 & $2 / 2$ \\
\hline
\end{tabular}


due to the similarity of Pf with $P$. berghei, but its molecular structure should be checked. It is in accordance with the fact that in P. falciparum malaria, the immunity induced by irradiated sporozoites is species-dependent yet it is cross-protective against different parasite strains [23]. Pv and Pf antigens were used due to unavailability of $P$. berghei antigen. This is done based on the finding that even thought early human studies in one volunteer suggested species specificity, but recent studies with rodent models have showed cross-species protection [24, 25]. There were positive results in some serum to Pv247 and Pv210 antigens. There was low effect of booster to the challenge that were done 2-4 weeks post booster. From these, it was known that injection with irradiation-attenuated sporozoites of $P$. berghei conferred low or no protection against live sporozoite challenge, even though it was supported by booster injected two weeks after the first injection.

Free radicals induced by irradiation may cause breaks in the protein chains or changes in their secondary or tertiary structure, resulting in modifications of their physicochemical properties such as fragmentation, crosslinking and aggregation. However, the knowledge on the alteration of protein profile of parasites after irradiation is still limited and it is needed to study the vaccine efficacy. Result of protein profiling of gamma ray irradiated sporozoites of $P$. berghei showed that there was a slight difference in the profiles of protein except for CSP (circumsporozoite protein) with molecular weight of $62 \mathrm{kDa}$ among doses of gamma rays of 0, 150, 175 and 200 Gy. However, the higher number and thicker bands (intensity) was seen in higher dose (200 Gy), showing the effectiveness of high dose of gamma rays in attenuating parasites (Fig. 3).

In this study with SDS-PAGE (sodium dodecyl sulfate-polyacrylamide gel electrophoresis), it was known that protein profiling of $P$. berghei infected salivary glands of Anopheles sp. showed four main polypeptide bands with the sizes of about 32, 40, 58 and $80 \mathrm{kDa}$. Besides that, in this experiment there was no separation between lateral and distal parts of salivary glands which has various proteins with different function and profiles. The main proteins were seen at the molecular weights of $62 \mathrm{kDa}$ and $110 \mathrm{kDa}$. It seems that protein of $110 \mathrm{kDa}$ was only seen at irradiation dose of $200 \mathrm{~Gy}$. These results provide basic informa-tion that would lead to further study on the role of sporozoite proteins in malaria vaccine development.

In another experiment, a band with molecular weight of $62 \mathrm{kDa}$ that corresponds to circumsporozoites protein was prominent and detected only in 150 and 175 irradiated salivary glands, suggesting the effect of gamma irradiation to the profile of sporozoite protein that depending on the dose of irradiation (Fig. 4). Again,

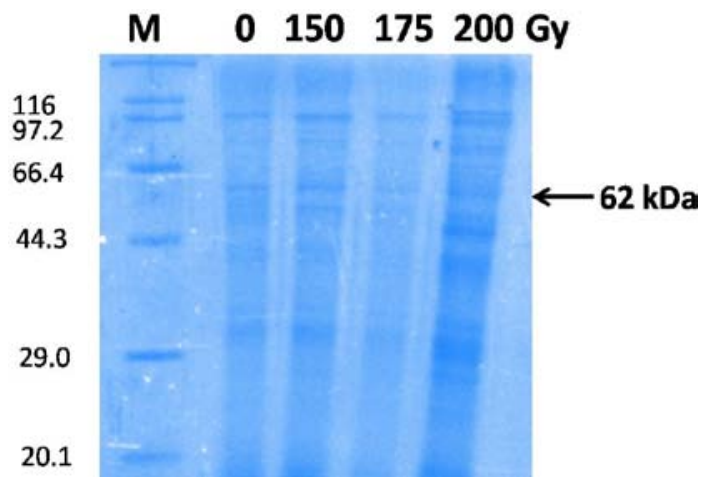

Fig. 3 Protein profile of irradiated sporozoites of $P$. berghei (molecular weight of $62 \mathrm{kDa}$ ) with SDS-PAGE.

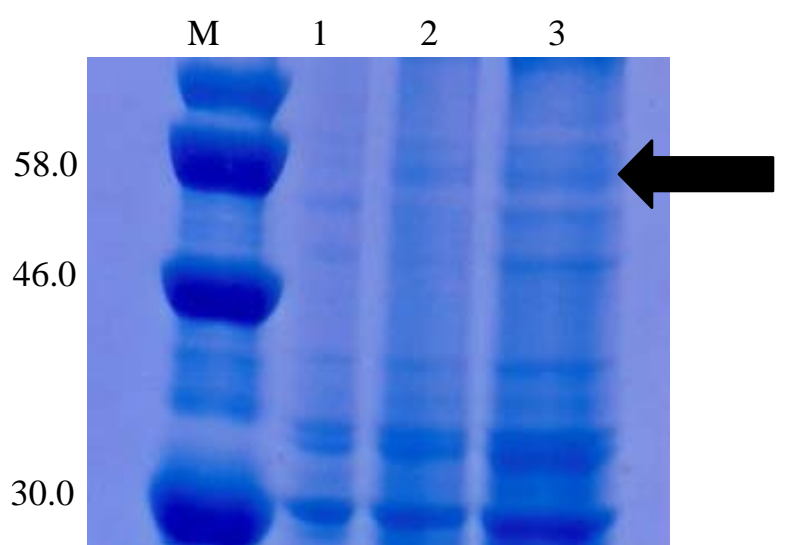

Fig. 4 Protein profile of irradiated sporozoites of $\boldsymbol{P}$. berghei with SDS-PAGE where a protein with molecular weight of $62 \mathrm{kDa}$ (arrow) was detected only in irradiated salivary glands. M, marker; lanes 1, 0; 2, $150 \mathrm{~Gy}$; and 3, 175 Gy. No available data for 200 Gy. 
the higher dose resulted in thicker bands where the intensity of several other subunit bands were increased as results of gamma radiation and reached maximum intensity at 175 Gy. There was no increase in bands associated with low molecular weight fragments (below $30 \mathrm{kDa}$ ) with the increase in the irradiation dose. Radiation may dissociate these protein fractions to small subunits and rearrangement to form a complex protein even high or small molecular weight compounds. Thus, the changes in protein profile were depended on radiation dose. To our opinion, this is the first report on the mosquito's salivary gland protein profiling after irradiation.

\section{Discussion}

In this report, we present our simple but valuable results on immune response in mouse blood post gamma rays attenuated whole parasites of $P$. berghei injection for malaria vaccine development. As compared to developing vaccines against viruses and bacteria, developing a vaccine against malaria is complicated by the complexity of the parasite as well as the host's response to the parasite. Host genetic variations play a significant role in conferring predisposition to malaria infection and determine the outcome of interactions between host and pathogen. Genetic variation in malaria parasite is central to the pathogenesis of the organism, as allelic variability in different clones is thought to facilitate immune evasion [26]. Based on these facts, it urgently needed a malaria vaccine that is specific for Indonesian people. To realize this expectation, this preliminary study was conducted by using murine malaria models to understanding the nature of biological responses and the role in development of immunity to malaria.

Injection with radiation-attenuated sporozoites uniquely leads to long lasting sterile immunity against malaria infection. In this experiment, we obtained the similar results, even though only in limited data. Complete protection against malaria in humans and mice can be obtained by inoculation with irradiated
Plasmodium sporozoites which infect hepatocytes in vivo as normal sporozoites, but they do not progress further to blood-stage infections and, therefore, do not induce malaria-associated pathology [20, 27, 28].

The major target of pre-eryrthrocytic vaccine development is the high numbers of sporozoites obtained from mosquitoes. As discussed partly in sentences above, to do that some factors contributes such as the susceptibility of mosquito to parasites. Parasites must complete a complex life cycle in mosquitoes in order to be transmitted from human to human. Vectors must be able to support parasite development through several key stages over 8 days to 15 days. Temperature changes will also shorten the development time of parasites in mosquitoes. Thus, geographic distribution of malaria is confined within climates favoring its extrinsic cycle, provided that other conditions do not limit mosquito survival. In turn, duration of the sporogonic development of malaria parasites in mosquitoes (Fig. 2) is an important component of vectoral capacity that measures the potential rate of contact between infectious vectors and susceptible hosts [29].

Among the practical applications of radiobiological techniques that may be of considerable interest for public health is the use of ionizing radiation in the preparation of vaccines. Radiation attenuated vaccines have been demonstrated to be an effective means of controlling certain parasitic infections such as malaria. Irradiation is a technically simple process that retains structural features of the microbial pathogen without destroying the natural antigens or the intrinsic adjuvants. Therefore, a strong immune response is induced in the vaccinated host. Irradiation destroys nucleic acid, making the microorganism unable to replicate so it can not establish an infection, but some residual metabolic activity may survive, so the irradiated microorganism can still find its natural target in the host. The successful use of irradiated plasmodium as a vaccine depends on finding a radiation dose which will significantly reduce the 
pathogenic effect of the larvae without seriously impairing their immunogenic power [30].

The dose of irradiation used to attenuate plasmodium is a most crucial factor. Therefore, to determine the dose of gamma-irradiation which will produce attenuated parasites without affecting the pre-erythocytic stabilities is the ultimate goal of many studies on irradiation vaccine. It is thought that the amount of radiation required to render the parasites non-viable is about $150 \mathrm{~Gy}$ for an inoculum of certain number of parasites. There is also evidence that exoerythrocytic stages may be more susceptible to gamma-rays than are blood parasites. It means that a most important step is to determine the minimum dose of irradiation required to adequately attenuate each sporozoite. To ensure that the parasites are sufficiently weakened for the vaccine, yet remain alive, they must be exposed to a radiation dose of at least $150 \mathrm{~Gy}$, but not much more. The researchers have revealed that to be safe for human trials, all mosquitoes must get their minimum radiation dose of $150 \mathrm{~Gy}$. It is only at this dose that they can be sure that the parasites are weak enough for the vaccine, but are still alive [31]. Our experiment on blood (erythrocytic) stage parasites also revealed that this dose of irradiation was most effective dose for attenuating the parasites to obtain vaccine material [32].

In this experiment, as the main target, radiation exposure is intended to inactivate parasites. According to the category of biological agents of radiological protection, the irradiation used in this vaccine materials development is the deterministic effects (harmful tissue reactions) due to large part of the killing/malfunction of cells following high doses (more than $100 \mathrm{~Gy}$ ) rather than stochastic effects. And the induction of tissue reactions is generally characterized by a threshold dose. The reason for the presence of this threshold dose is that radiation damage (serious malfunction or death) of a critical population of parasite cells. So that at high doses radiation exposures may cause deterministic effects (tissue reactions). The extent of damage depends upon the absorbed dose and dose rate as well as radiation quality and the sensitivity of the target materials [31, 33].

Here, we sought the most effective dose of irradiation to atenuate parasites through protein profiling. Radiation is known to cause disintegration and aggregation of protein molecule and this is predicted occured in the present study. Gamma radiation is generally attributed to direct breakdown in protein where the irradiation dose used considered very important factor. Radiation may also dissociate these protein fractions to small subunits and rearrangement to form a complex protein even high or small molecular weight compounds. The direct breakdown of the molecule by ionization capacity of the passing photon may be one possibility. In addition, radiation normally cause the formation of disulphide bridge between polypeptide chain which may be effect on the aggregation of the low molecular weight protein and effect on its conformation [34]. Thus, irradiation produces some chemical changes, which lead to the production of small amounts of radiolytic products that may cause breaks in the protein chains or changes in their secondary or tertiary structure, resulting in modifications of their physicochemical properties, namely, fragmentation, crosslinking and aggregation [35]. These changes were confirmed by SDS-PAGE.

\section{Conclusions}

The experiments showed that irradiated sporozoite as vaccine material had a low effectivity or did not seem in eliciting immune response upon booster and challenge. The low capacity of An. farauti as the most potential vector in our experiment affecting the number of sporozoites produced in the salivary glands. There was no alteration of protein profile of sporozoite infected salivary glands post gamma ray irradiation, indicating the effectiveness of irradiation.

\section{Acknowledgment}

This research in part was conducted under Incentive 

of Plasmodium berghei

Research Project No. RD-2009-0047 from the Indonesian Ministry of Research and Technology.

\section{References}

[1] World Malaria Report 2010, World Health Organization, Geneva, 2011.

[2] C. Delacollette, C. D’Souza, E. Christophel, K. Thimasarn, R. Abdur, D. Bell, et al., Malaria trends and challenges in the Greater Mekong Subregion, Southeast Asian J. Trop. Public Health 40 (4) (2009) 674-691.

[3] Directorate General of Disease Control and Environmental Health, Malaria, 2010, http://www.pppl.depkes.go.id/images_data/MA LARIA.pdf.2010 (accessed June 23, 2013).

[4] Anonymous, Epidemiology of Malaria in Indonesia, Buletin of Data Window and Health Information, Ministry of Health of Republic of Indonesia, 2011.

[5] M.F. Good, The hope but challenge for developing a vaccine that might control malaria, European Journal of Immunology 39 (4) (2009) 939-943.

[6] D.F. Clyde, Immunization of man against falciparum and vivax malaria by use of attenuated sporozoites, Am. J. Trop. Med. Hyg. 24 (3) (1975) 397-401.

[7] V. Nussenzweig, R.S. Nussenzweig, Development of a sporozoite malaria vaccine, Mem. Inst. Oswaldo Cruz Rio de Janeiro 81 (Suppl. II) (1986) 45-55.

[8] N. Yoshida, R.S. Nussenzweig, P. Potocnjak, V. Nussenzweig, M. Aikawa, Hybridoma produces protective antibodies directed against the sporozoite stage of malaria parasite, Science 207 (4426) (1980) 71-73.

[9] M.J. Stewart, R.J. Nawrot, S. Schulman, J.P. Vanderberg, Plasmodium berghei sporozoite invasion is blocked in vitro by sporozoite-immobilizing antibodies, Infect. Immun. 51 (3) (1986) 859-864.

[10] A.A. Sultan, V. Thathy, U. Frevert, K.J. Robson, A. Crisanti, V. Nussenzweig, et al., TRAP is necessary for gliding motility and infectivity of plasmodium sporozoites, Cell 90 (3) (1997) 511-522.

[11] J.L. Komisar, Malaria vaccines, Front Biosci. 12 (2007) 3928-3955.

[12] B. Genton, Z.H. Reed, Asexual blood-stage malaria vaccine development: Facing the challenges, Curr. Opin. Infect. Dis. 20 (5) (2007) 467-475.

[13] W.R. Ballou, M. Arevalo-Herrera, D. Carucci, T.L. Richie, G. Corradin, C. Diggs, et al., Update on the clinical development of candidate malaria vaccines, Am. J. Trop. Med. Hyg. 71 (2 Suppl) (2004) 239-247.

[14] S. Waki, J. Tamura, M. Imanaka, S. Ishikawa, M. Suzuki, Plasmodium berghei: Isolation and maintenance of an irradiation attenuated strain in the nude mouse, Exp. Parasitol. 53 (3) (1982) 335-340.
[15] B.T. Wellde, R.A. Ward, R. Ueoka, Aspects of immunity in mice inoculated with irradiated Plasmodium berghei, Mil. Med. 134 (10) (1969) 1153-1164.

[16] S. Waki, I. Yonome, M. Suzuki, Plasmodium yoelii: Induction of attenuated mutants by irradiation, Exp. Parasitol. 62 (3) (1986) 316-321.

[17] J. Rivero, L. Urdaneta, N. Zoghbi, M. Pernalete, Y. Rubio-Palis, F. Herrera, Optimization of extraction procedure for mosquito DNA suitable for PCR-based techniques, International Journal of Tropical Insect Science 24 (3) (2004) 266-269.

[18] S. Paulie, P. Perlmann, H. Perlmann, Enzyme-linked immunosorbent assay, in: J.E. Celis (Ed.), Cell biology: A Laboratory Handbook, 3rd ed., Elsevier Science, 2003.

[19] U.K. Laemmli, Cleavage of structural proteins during the assembly of the head of bacteriophage T4, Nature 227 (1970) 680-685.

[20] C. Boudin, J. Lyannaz, M.F. Bosseno, J. Chaize, P. Carnevale, Production of sporozoites of human Plasmodium in Bobo-Dioulasso, Burkina Faso, Ann. Soc. Belg. Med. Trop. 69 (1) (1989) 3-23.

[21] H.G. Sarah, Factors affecting Plasmodium falciparum sporozoite formation in Anopheles mosquitoes, Ph.D. Thesis [Online], University of Glasgow, 2010, http://theses.gla.ac.uk/1750/1/2010humphreysphd.pdf (accessed June 6, 2014).

[22] O. Silvie, J.P. Semblat, J.F. Franetich, L. Hannoun, W. Eling, D. Mazier, Effects of irradiation on Plasmodium falciparum sporozoite hepatic development: Implications for the design of pre-erythrocytic malaria vaccines, Parasite Immunology 24 (4) (2002) 221-223.

[23] S.A. Mikolajczak, A.S. Aly, S.H. Kappe, Pre-erythrocytic malaria vaccine development, Curr. Opin. Infect. Dis. 20 (5) (2007) 461-466.

[24] M. Sedegah, W.W. Weiss, S.L. Hoffman, Cross-protection between attenuated Plasmodium berghei and Plasmodium yoelii sporozoites, Parasite Immunol. 29 (11) (2007) 559-565.

[25] M. Mauduit, A.C. Gruner, R. Tewari, N. Depinay, M. Kayibanda, J.M. Chavatte, et al., A role for immune responses against non-CS components in the cross-species protection induced by immunization with irradiated malaria sporozoites, PLoS One 4 (11) (2009) e7717.

[26] B.A. Biggs, L. Gooze, K. Wycherley, W. Wollish, B. Southwell, J.H. Leech, et al., Antigenic variation in Plasmodium falciparum, Proceeding of the National Academy of Sciences USA 88 (1991) 9171-9174.

[27] R.S. Nussenzweig, C.A. Long, Malaria vaccines: Multiple targets, Science 265 (5177) (1994) 1381-1383.

[28] A. Suhrbier, L.A. Winger, E. Castellano, R.E. Sinden, Survival and antigenic profile of irradiated malarial sporozoites in infected liver cells, Infect. Immun. 58 (9) 
(1990) 2834-2839.

[29] C. Dye, The analysis of parasite transmission by bloodsucking insects, Annu. Rev. Entomol. 37 (1992) 1-19.

[30] Anonymous, Using gamma radiation preserves T-cell responses in bacterial vaccine, Medical Research News [Online],

http://health.ucsd.edu/news/2006/07_25_Raz.htm (accessed July 26, 2006).

[31] Anonymous, Radiation Weakened Parasites: Possible New Malaria Vaccine? http://www.medindia.net/news/Radiation-Weakened-Para sites-Possible-New-Malaria-Vaccine-29169-1.htm (accessed November 10, 2007).

[32] M. Syaifudin, D. Tetriana, Darlina, S. Nurhayati, The feasibility of gamma irradiation for developing malaria vaccine, Atom Indonesia Journal 37 (3) (2011) 91-101.

[33] F.H. Attix, Introduction to Radiological Physics and Radiation Dosimetry, Wiley, New York, 1986.

[34] H.S. El-Beltagi, O.K. Ahmed, W. El-Desouky, Effect of low doses $\gamma$-irradiation on oxidative stress and secondary metabolites production of Rosemary (Rosmarinus officinalis L.) callus culture, Radiat. Phys. Chem. 80 (9) (2011) 968-976.

[35] S. Celik, A. Basman, E. Yalcin, H. Koksel, Effects of gamma irradiation on protein electophoretic properties, water absorption and cooking quality of bean and chickpea, in: Proceedings of 2001 AACC Annual Meeting, Charlotte, North Carolina, USA, October 16-19, 2011. 